Supplementary Materials for

\title{
Genome hypermobility by lateral transduction
}

John Chen, Nuria Quiles-Puchalt, Yin Ning Chiang, Rodrigo Bacigalupe, Alfred Fillol-Salom, Melissa Su Juan Chee, J. Ross Fitzgerald, José R Penadés.

$\begin{array}{lll}\text { Correspondence to: } & \text { John Chen: } & \text { miccjy@nus.edu.sg } \\ & \text { José R Penadés: } & \text { JoseR.Penades@glasgow.ac.uk }\end{array}$

This PDF file includes:

Materials and Methods

Figs. S1 to S18

Tables S1 to S4 


\section{Materials and Methods}

\section{Bacterial strains and growth conditions}

Bacterial strains used in these studies are listed in Table S1. S. aureus strains were grown in tryptic soy broth (TSB) and TSB agar. E. coli strains were grown in Luria-Bertani (LB) broth or on LB agar plates. Antibiotic resistant $S$. aureus were selected and maintained on 5 $\mu \mathrm{g} \mathrm{ml} l^{-1}$ erythromycin, $10 \mu \mathrm{g} \mathrm{ml}^{-1}$ chloramphenicol, $300 \mu \mathrm{g} \mathrm{ml}^{-1}$ streptomycin, $5 \mu \mathrm{g} \mathrm{ml}^{-1}$ tetracycline, $1.0 \mathrm{mM} \mathrm{NaAsO}_{2}$, or $0.1 \mathrm{mM} \mathrm{CdCl}_{2}$. Antibiotic resistant $E$. coli were selected and maintained on ampicillin, $100 \mu \mathrm{g} \mathrm{ml}^{-1}$.

\section{DNA methods}

The oligonucleotides and plasmids used in this study are listed in Tables S2 and S3, respectively. The sequences of all phages and strains in this article were previously available in GenBank. Plasmid DNA preparation and agarose gel purification kits were purchased from Qiagen. Oligonucleotides were purchased from Integrated DNA Technologies. 1st Base (Singapore) or the University of Glasgow core facilities performed DNA sequencing. Phusion polymerase, restriction enzymes, and ligase were purchased from New England Biolabs.

\section{Whole genome sequencing}

Bacterial cultures were grown and at indicated time points after MC-treatment $4 \mathrm{ml}$ of sample was taken for DNA extraction using GenElute Bacterial Genomic DNA kit (Sigma) following the manufacturer's instructions. DNA samples were quality control tested using Agilent Bioanalyzer 2100 at the University of Glasgow, Polyomics Facility. Whole genome sequencing (WGS) was performed at the University of Glasgow Polyomics Facility using Illumina NextSeq 500 obtaining 75 bp pair end reads with no initial PCR amplification step. Trimmed reads were mapped to the appropriate phage genome: 80a (NC_009526.1), $\$ 11$ 
(NC_004615.1), фNM1 (DQ530359.1), фNM2 (DQ530360.1), фNM3 (NC_008617.1), фNM4 (DQ530362.1), ф52a (NC_007062.1).

\section{Total RNA extraction and mRNA enrichment}

Bacterial cultures were grown and at indicated time points after MC-treatment samples for RNA extraction were taken and mixed with two volumes of RNA protect reagent (QIAgen) incubated for 5 minutes at room temperature and cell pellets harvested by centrifugation. The bacterial pellet was resuspended in $1 \mathrm{ml}$ of TRIzol reagent (Ambion) and was lysed in a FastPrep-24 homogenizer (MP Biomedicals) using two cycles of $60 \mathrm{~s}$ at $6.5 \mathrm{~m} \mathrm{~s}^{-2}$ interrupted by a 5 minutes incubation on ice. Total RNA was extracted using the Ambion PureLink Trizol Plus kit according to the manufacturer's instructions. Genomic DNA was removed using oncolumn DNase digestion step using the RNase-free DNase kit (Qiagen) and residual DNA was removed by a second DNase treatment using RQ1 DNase (Promega). Total RNA samples were enriched for mRNA using MICROBexpress mRNA enrichment kit (Ambion). Experiments were performed in triplicate. Samples for RNA-seq analysis were quality control tested for mRNA enrichment using Agilent Bioanalyzer 2100 at the University of Glasgow, Polyomics Facility.

\section{RNA-seq transcriptome analysis}

cDNA synthesis and sequencing was performed at the University of Glasgow Polyomics Facility using Illumina NextSeq 500 obtaining 75 bp single end reads. Sequencing reads for each of the libraries were mapped to the appropriate reference genomes: $80 \alpha$ (NC_009526.1), ф11 (NC_004615.1), фNM1 (DQ530359.1), фNM2 (DQ530360.1), ф52a (NC_007062.1) using the RNA-Seq pipeline READemption (27). The coverage subcommand of this software was used to produce strand specific coverage counts normalized by 100,000 division. Strands coverage values of the phage spanning regions for different replicates were plotted with values $>1 \log 10$ corrected. 


\section{Allelic Exchange}

For the generation of insertions in the $S$. aureus chromosome, allelic exchange was performed as previously described (28-30).

\section{Phage transductions}

Preparation of phage lysates, transduction, and titrations were performed as previously described $(28,29,31,32)$. Briefly, for phage lysates, lysogens were grown to mid log in TSB, normalized by $\mathrm{OD}_{600}$, and adjusted to $2 \mu \mathrm{g} \mathrm{ml}^{-1}$ Mitomycin $\mathrm{C}$ (Sigma) until complete Iysis; lysates by infection were made by infecting the same density of cells with an $\mathrm{MOI}$ of 0.1 until complete lysis. Lysates were then adjusted to $1 \mu \mathrm{g} \mathrm{ml}^{-1}$ DNAse I and $1 \mu \mathrm{g} \mathrm{ml}^{-1}$ RNAase and filter sterilized $(0.2 \mu \mathrm{m}$ pore) before use. Phage titers were determined by plaque formation on RN450 using phage agar (31). For transductions, cells were infected for 30 minutes and then adjusted to $100 \mathrm{mM}$ sodium citrate, mixed with $3 \mathrm{ml}$ of top agar, and plated on selective agar. The results are reported as the transduction units (TrU) $\mathrm{ml}^{-1}$, divided by the corresponding plaque forming units (PFU) $\mathrm{ml}^{-1}$, and represented as the TrU of an average phage titer (1E+9 PFU).

\section{Marker co-transduction analysis}

Lysates from phage infection or lysogen induction of strains containing both a $\mathrm{Cd}^{\mathrm{R}}$ and a $\operatorname{Tet}^{R}$ marker at varying distances apart were used to determine marker co-transduction frequencies. $A$ Tet $^{R}$ marker at $+15 \mathrm{~kb}$ was not evaluated because marker expansion would push the marker too close to the headful demarcation, and it is predicted there would not be sufficient flanking DNA to support homologous recombination in the recipient. The $\mathrm{Cd}^{\mathrm{R}}$ transductants of three independent lysates (100 transductants each) were tested for Tet ${ }^{R}$ and the frequency represented as the $\left(\mathrm{Tet}^{\mathrm{R}} / \mathrm{Cd}^{\mathrm{R}}\right) \times 100 \%$. 


\section{Spontaneous transduction analysis}

Strains were grown to mid log in TSB and normalized by $\mathrm{OD}_{600}$. Equal numbers $(\sim 1 \mathrm{E}+9$ cells) of donor and recipient were adjusted to a final 1:1 mixture of TSB and phage buffer. For plating, a $500 \mu$ l equivalent of $\mathrm{OD}_{600}=1.0$ (to normalize for growth) was adjusted to 100 $\mathrm{mM}$ sodium citrate, mixed with $3 \mathrm{ml}$ of top agar, and plated on $\mathrm{TSA}+\mathrm{CdCl}_{2}+$ Streptomycin. The results are reported as the transduction units $(\mathrm{TrU}) \mathrm{ml}^{-1}$ equivalent of $1 \mathrm{ml}$ of $\mathrm{OD}_{600}=1.0$ to adjust for minor growth.

\section{Determination of the presence of defective $80 \alpha$ prophages in $80 \alpha$ lateral transductants}

Transductants from lysates of $80 \alpha$ prophage induction were tested for defective $80 \alpha$ prophages by growing $100 \mathrm{Cd}^{\mathrm{R}}$ transductants and challenging them with wt $80 \alpha$ phage. Lysis by $80 a$ suggests a lack of $80 a$ lysogeny, and lack of lysis suggests the strain is protected from superinfection by $80 \alpha$ lysogeny. In addition, the same $100 \mathrm{Cd}^{\mathrm{R}}$ transductants were further tested for $80 \alpha$ lysogeny by PCR analysis using primer pairs 80alpha$1 \mathrm{~m} / 80$ alpha-2c and 80alpha-3m/80alpha-4c specific to $80 \alpha$ (Table S2).

\section{Inducible complementation of the $\phi 52 a-f s$ mutant}

Lysogens of wt $\phi 52 a$ and $\phi 52 a-f s ~\left(r e p_{\phi 52 a}\right.$ frame-shift) were grown at $32^{\circ} \mathrm{C}$, normalized by $\mathrm{OD}$, and MC-induced ( $\mathrm{t}=0)$. Anhydrotetracycline $(31.25 \mathrm{ng} / \mathrm{ml})$ was added $0,30,60,90$, and 120 minutes for complementation by $\mathrm{P}_{\text {tet }}-$ rep $_{\phi 52 a}$. The cells were mechanically lysed 2 hours $\left(32^{\circ} \mathrm{C}, 100 \mathrm{rpm}\right)$ after the addition of anhydrotetracycline in a Precellys 24 (Bertin) with $0.1 \mathrm{~mm}$ Beadbug silica beads (Sigma-Aldrich). Physical disruption was done to harvest all lysates at fixed times because the $\phi 52 a-f s$ strains do not lyse on their own. Also, mechanical agitation with $0.1 \mathrm{~mm}$ silica beads does not detectably degrade phage titers. The lysates were then filter sterilized and treated as above. Plaque forming units were determined on a S. aureus host that constitutively expresses $r e p_{\phi 52 a}$ because the $\phi 52 a-f s$ mutant is unable to form plaques on RN450 alone. 


\section{Analysis of gene content, gene synteny and recombination}

Complete annotated genome sequences of $100 \mathrm{~S}$. aureus strains representative of the species diversity were downloaded from the NCBI database (Table S4). A core genome alignment of all strains was obtained using Roary with default parameters (33) and FastTree (34) was used to construct a Maximum Likelihood phylogenetic tree with a general timereversible (GTR) model of nucleotide substitution. Next, we used BLASTn to identify $1 \mathrm{~kb}$ sequences that included the gene most proximal to the Sa2 and Sa6 attB sites in the genome of $S$. aureus strain JKD6159, and extracted $140 \mathrm{~Kb}$ of nucleotide sequence upstream and downstream of this locus in selected strains using Bedtools (35). Genomic coordinates were manually inspected to ensure homologous regions were being analyzed before gene annotation using Prokka (36). Clusters of orthologous genes were identified using the OrthoMCL algorithm (37) from the open-source get_homologues software (38) with parameters $-\mathrm{C} 50 \%$, - S $30 \%$, $-\mathrm{F} 1$, before removal of paralogues. Easyfig (39) was used to visualize the genomic regions, and genes were coloured according to their conservation in the clusters identified. In order to analyze the impact of recombination on the flanking region of the Sa2 and $\mathrm{Sa} 6$ attB sites, the sequences of conserved clusters of genes (defined as being present in over $90 \%$ of the isolates) were aligned using MUSCLE (40). Subsequently, the individual alignments were concatenated into a super alignment using the software tool catfasta2phyml.pl, according to the gene order in the reference $S$. aureus strain JKD6159, before identification of recombinant regions with ClonalFrameML (41) using default parameters. 

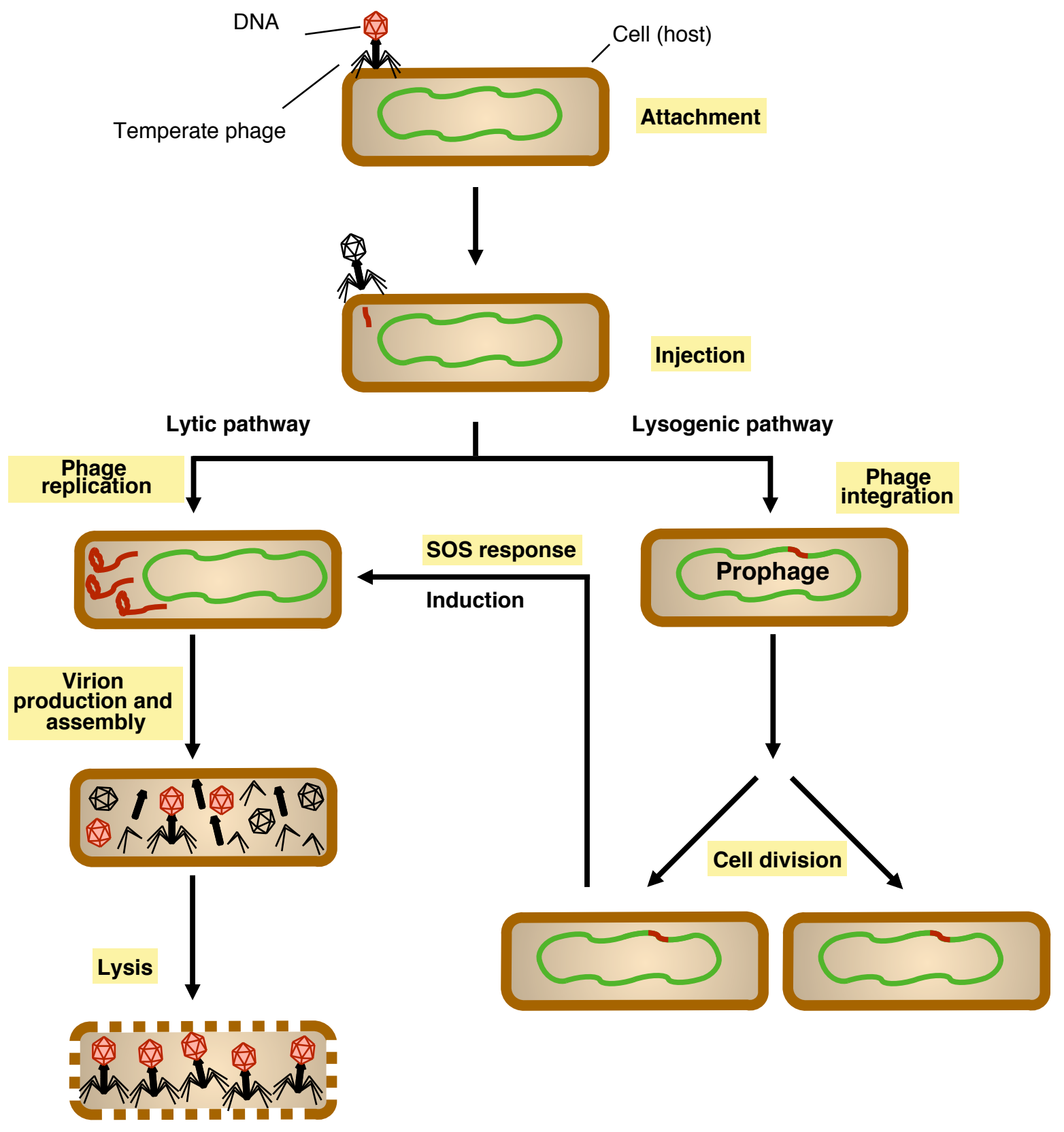

Figure S1. Lytic and lysogenic phage life cycles. 
A
Top strand
$\phi 11$
- Not induced
- $30^{\prime}$ after induction
- $60^{\prime}$ after induction

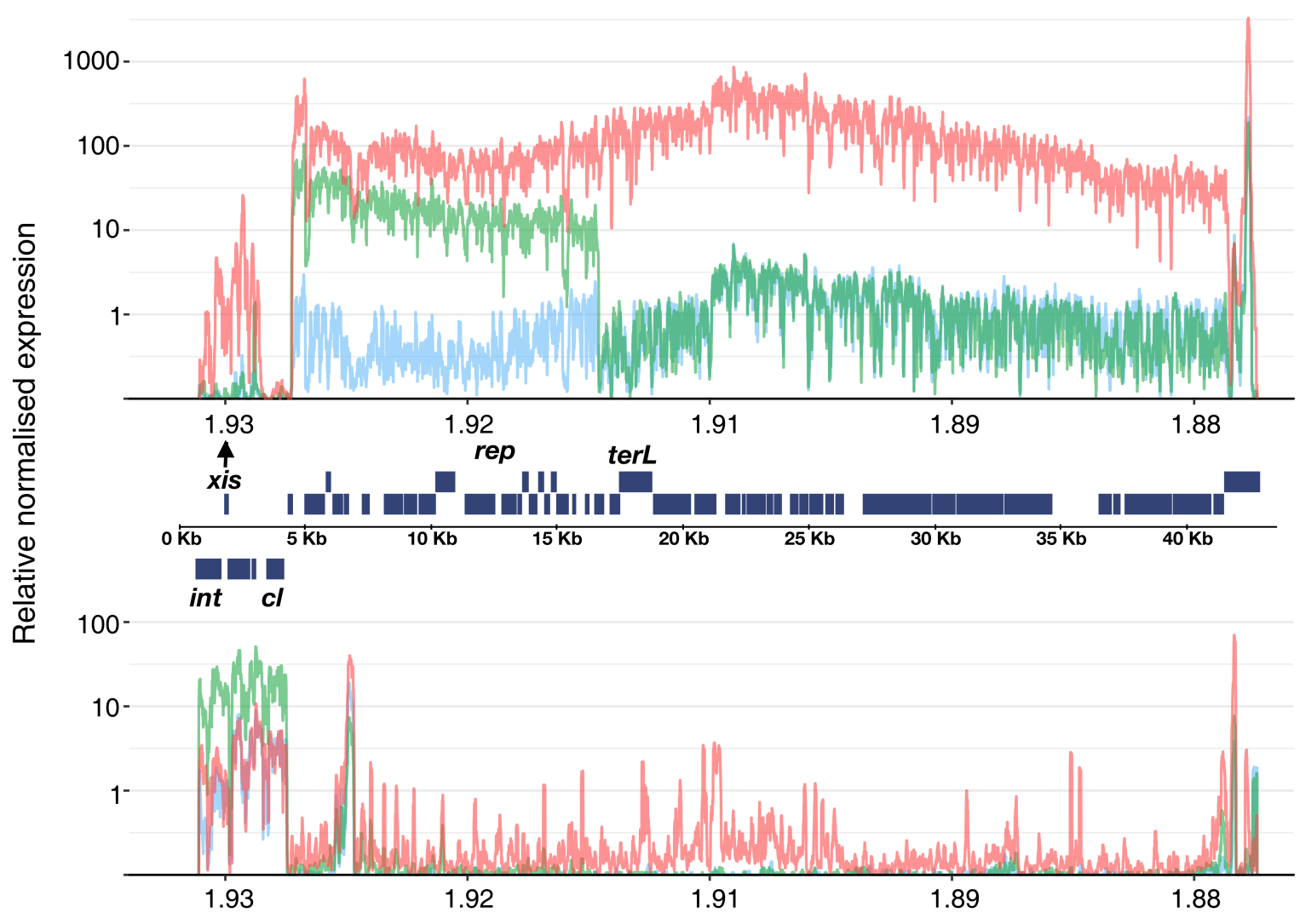

Bottom strand

Figure S2. Transcriptomic analyses for phages $\phi 11, \phi N M 1$, and $\phi N M 2$.

Transcriptional analysis showing expression of the early and late genes from phages (A) $\phi 11$, (B) $\phi N M 1$, and (C) $\phi N M 2$. Strains RN451 ( $\phi 11)$ or Newman ( $\phi N M 1$ and $\phi N M 2)$ were induced with mitomycin $\mathrm{C}$ and samples were analyzed without induction (light blue) or at 30 (early genes, green) and 60 minutes (late genes, red) after induction. 
B

Top strand

\section{$\phi N M 1$}

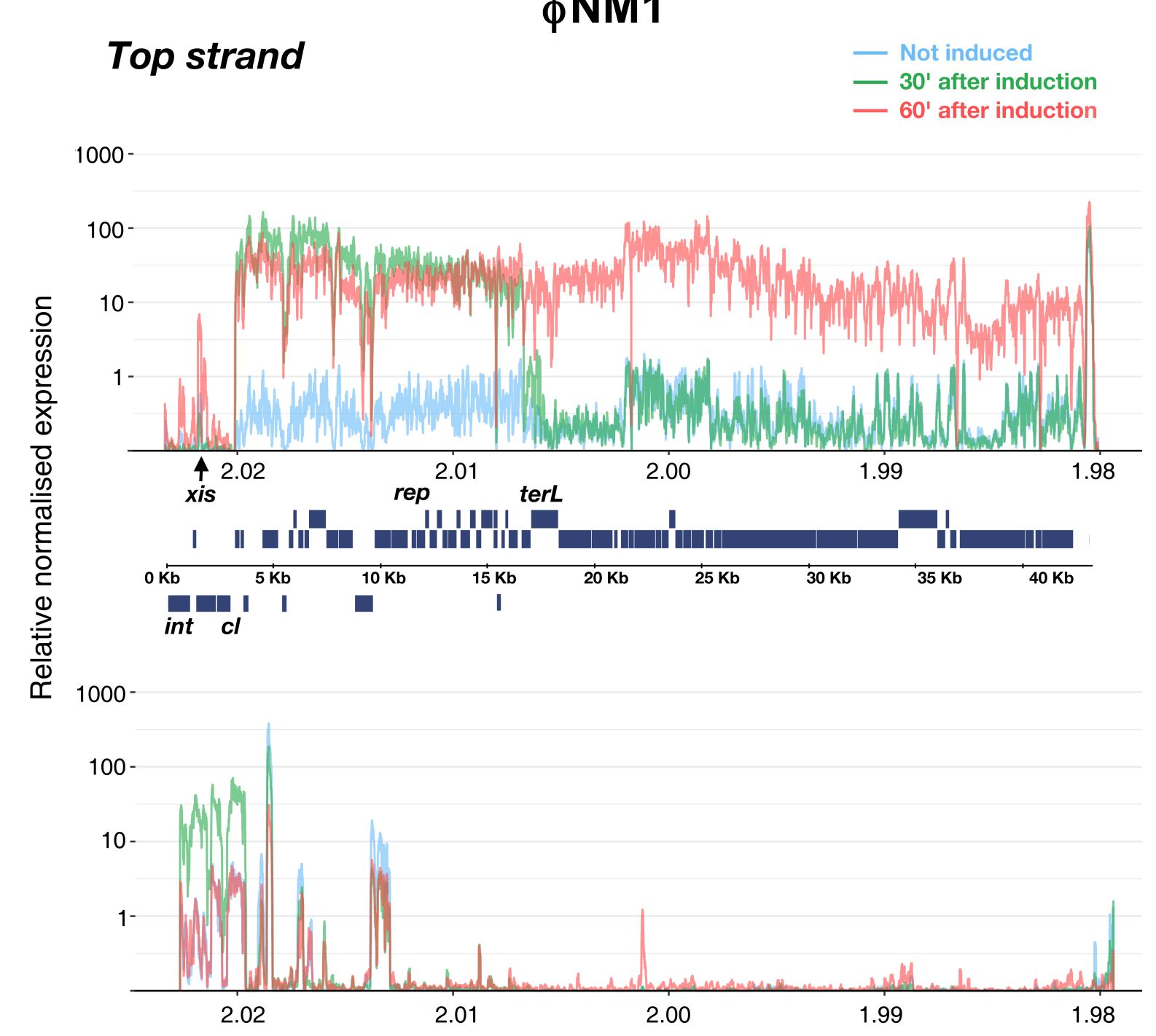

\section{Bottom strand}


C фNM2

Top strand

- Not induced

- 30' after induction

- 60' after induction

$1000-$
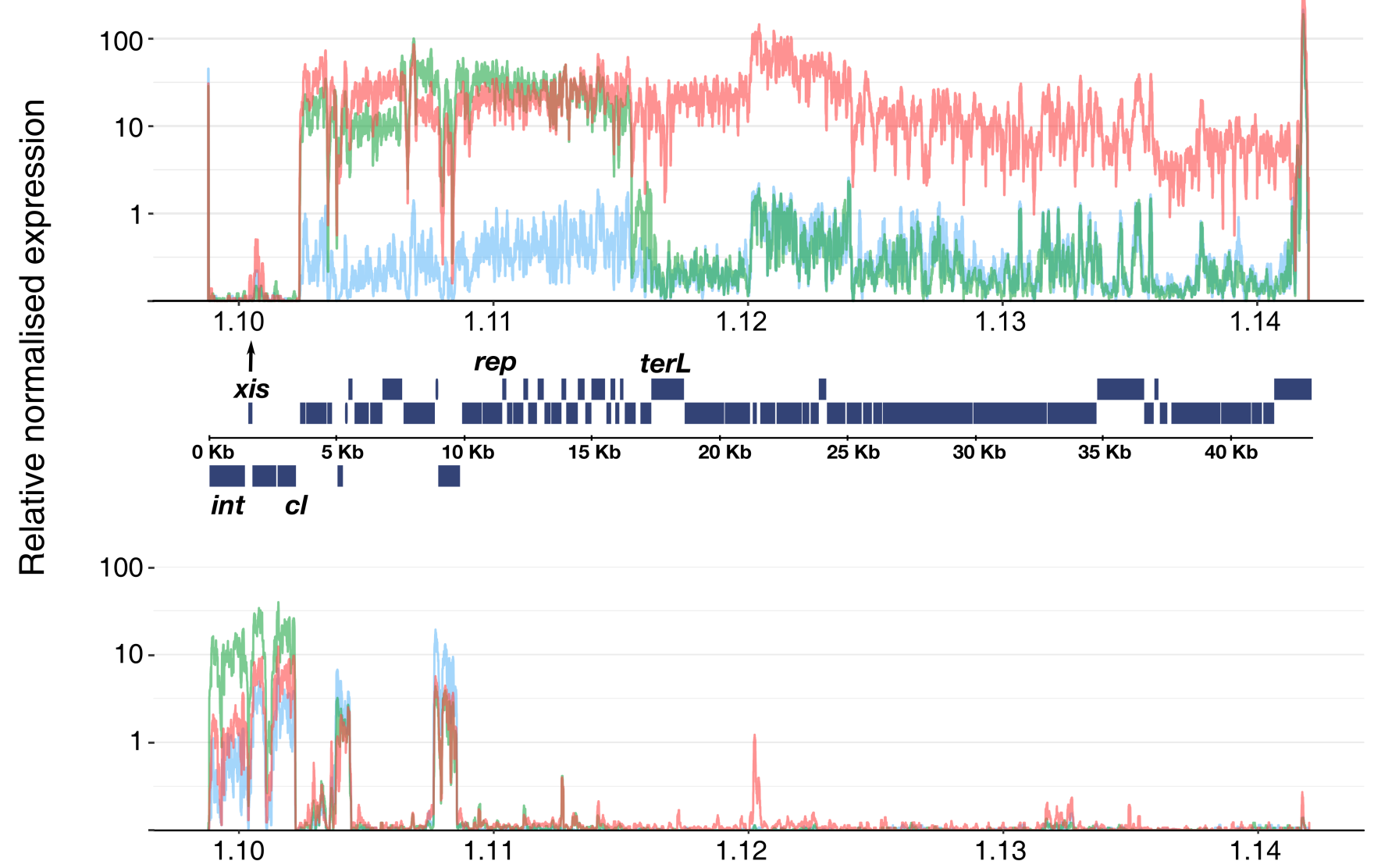

Bottom strand 


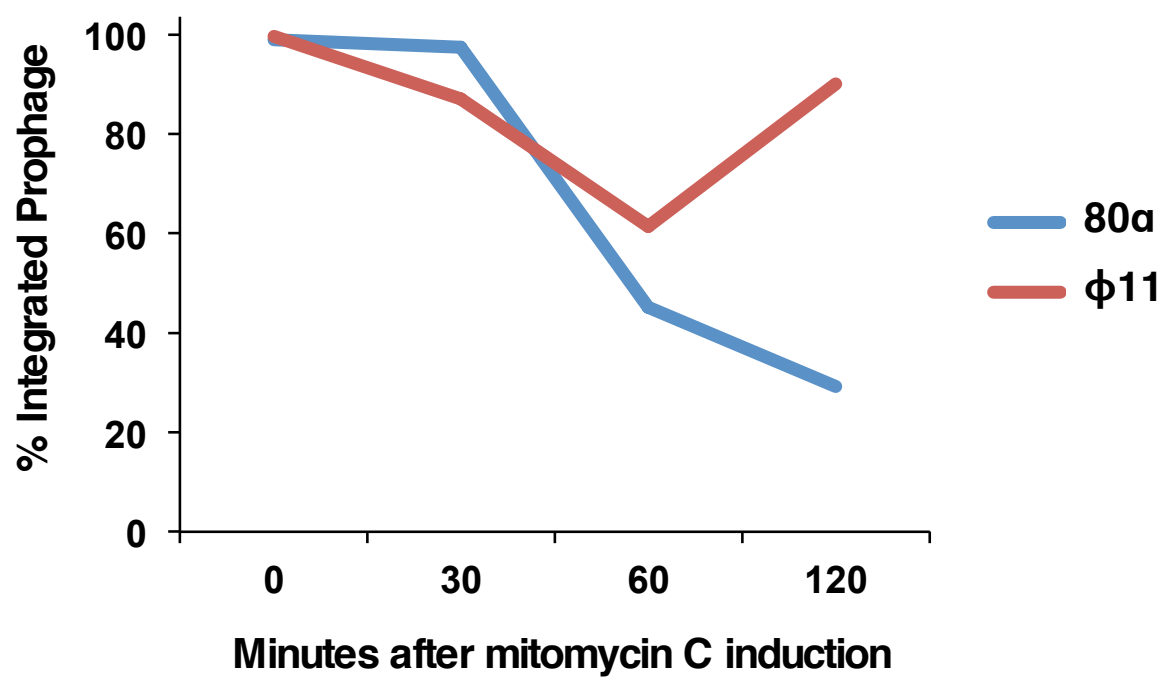

Figure S3. Prophages excise late from the bacterial chromosome.

Percentage of reads spanning the chromosome-attL phage region (integrated phage) out of the total (chromosome-attL phage plus chromosome-chromosome). 


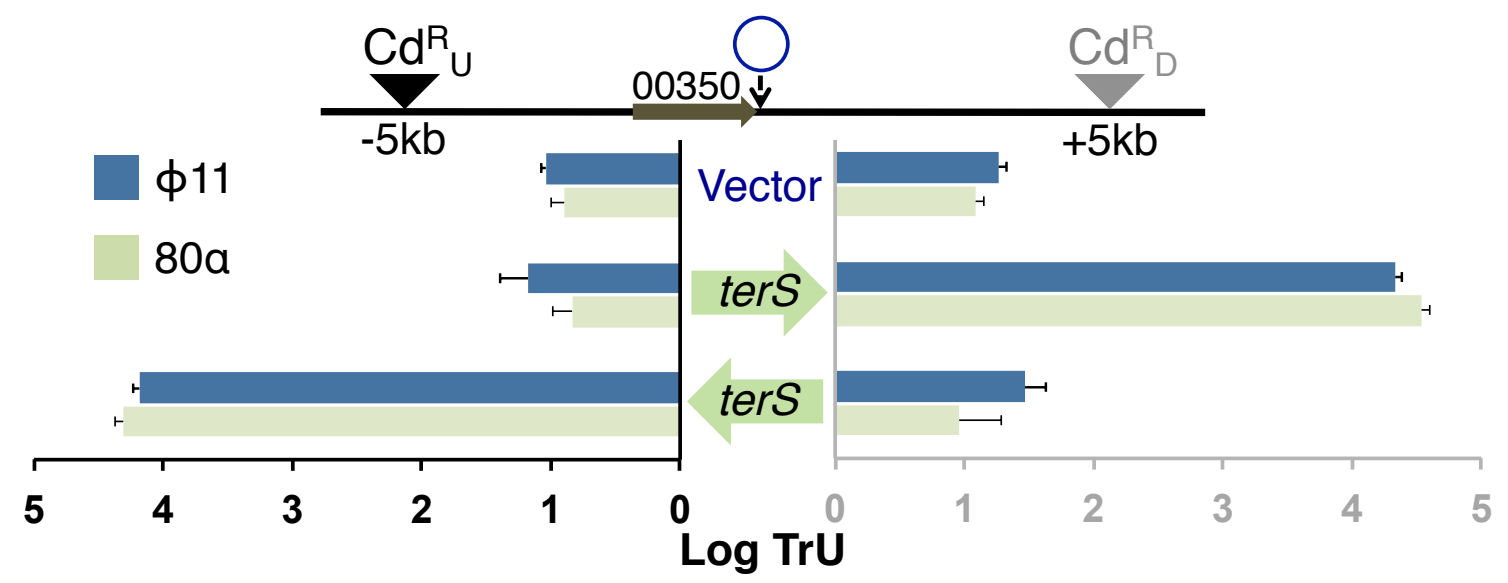

Figure S4. Determination of a pac site in terS $S_{i 11}$ and its directionality of packaging.

Phage pac sites are commonly embedded in structural terS genes, and to determine if the same follows for the staphylococcal phages we inserted the $\phi 11$ terS gene $\left(\operatorname{ter} S_{\phi 11}\right)$ at an unlinked site (SaPI 4 attB) in RN450, using a single-copy integration vector flanked by either a cadmium marker $5 \mathrm{~kb}$ upstream $\left(\mathrm{Cd}^{\mathrm{R}} \mathrm{u}\right)$ or downstream $\left(\mathrm{Cd}^{\mathrm{R}}{ }_{\mathrm{D}}\right)$. Since $80 \alpha$ and $\phi 11$ carry nearly identical terS genes, ter $S_{\phi 11}$ was used for both phages. These strains were infected with phage and the resulting phage lysates were tested as donors of cadmium resistance to S. aureus. Fig. S4 shows that the transfer frequencies were three orders of magnitude greater than vector alone, only when the ter $S_{\phi 11}$ gene was oriented such that the 3 ' end was closer to the marker, showing that the $\operatorname{ter} S_{\phi 11}$ gene encodes the bona fide $\phi 11$ pac site that directs unidirectional packaging toward the 3 ' end of the gene. 


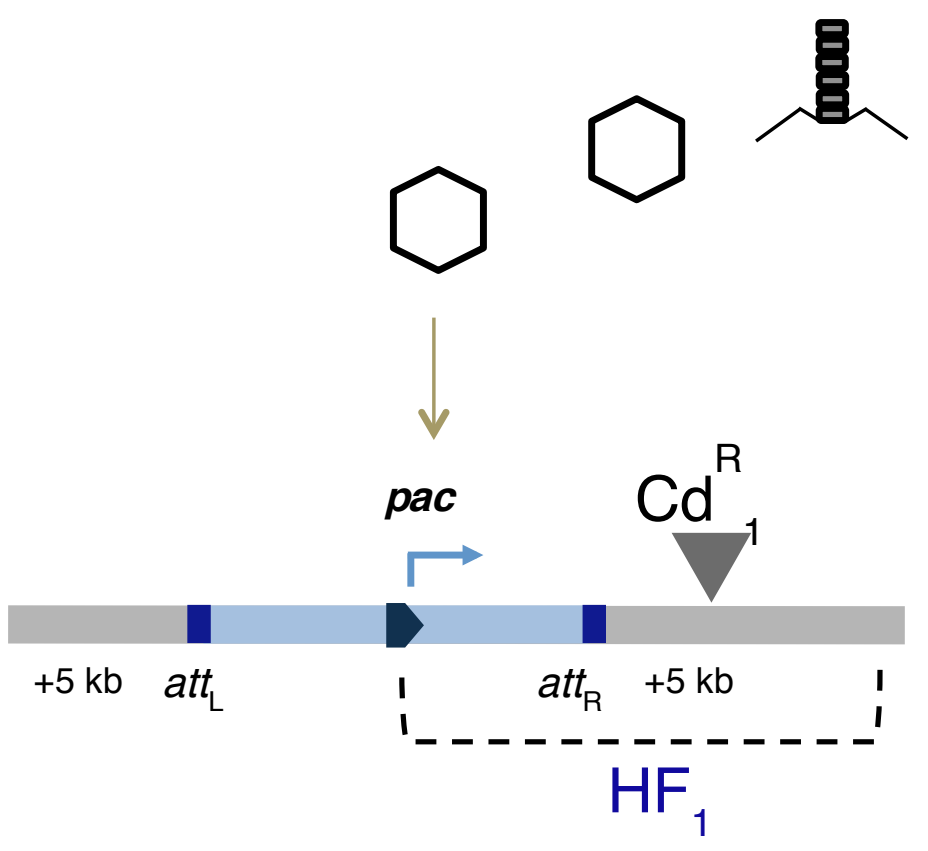

Figure S5. Model for gene transfer resulting from in situ packaging after prophage induction.

Location of the $C d^{R}{ }_{1}$ adjacent to the phage $\phi 11$ attB site. 
A

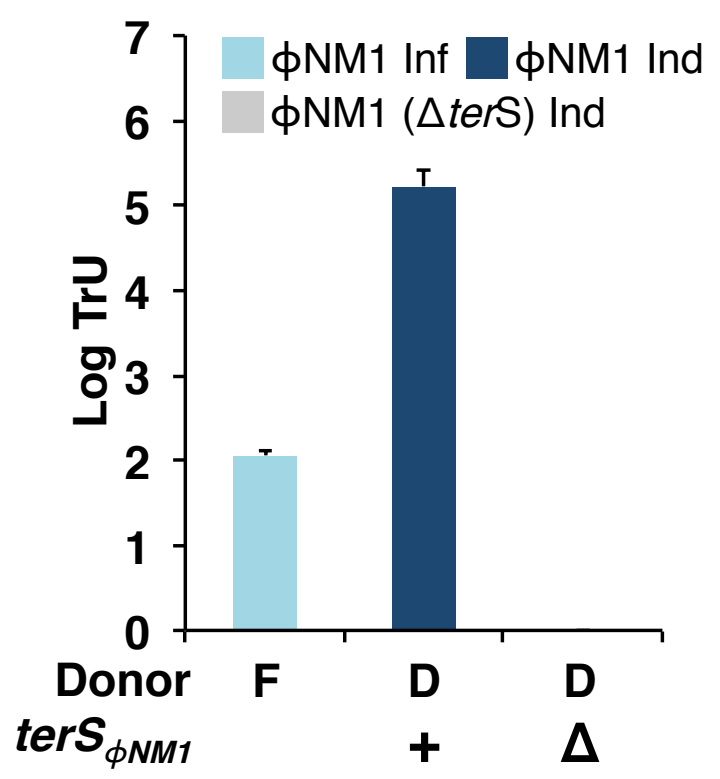

B

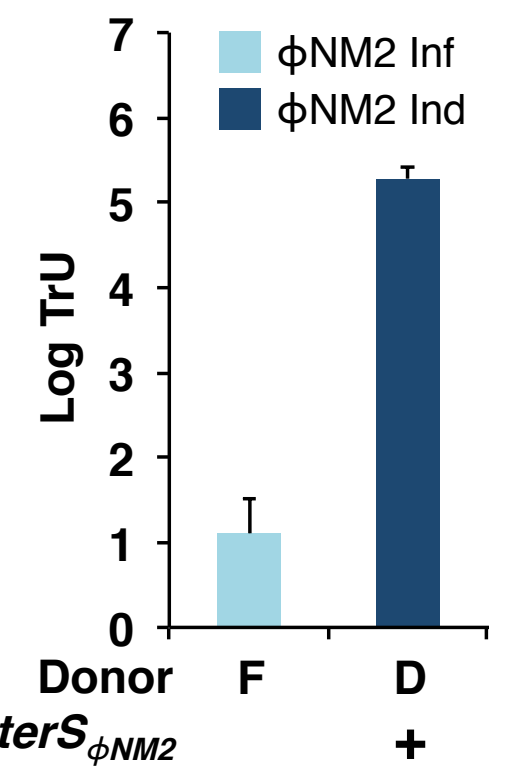

Figure S6. Staphylococcal phages and lateral transduction.

(A) фNM1 infection or lysogen induction tested for transfer of a $\mathrm{Cd}^{\mathrm{R}}{ }_{1} 5 \mathrm{~kb}$ downstream of the $a_{t t B_{\phi N M 1}}$ (same as $\left.a t t B_{\phi 11}\right)$ (B) $\phi N M 2$ infection or lysogen induction tested for transfer of a $\mathrm{Cd}^{\mathrm{R}} 4 \mathrm{~kb}$ downstream of the $a_{t t B_{\Phi N M 2}}$ (same as attB ${ }_{80 \alpha}$ ). Non-lysogenic strains (light blue) were infected $(F)$ or the lysogenic wt $(+$, dark blue) and terS deletion ( $\Delta$, gray) strains were induced (D) with mitomycin $\mathrm{C}$ and the lysates tested for transduction into $S$. aureus. Transduction units (TrU) $\mathrm{ml}^{-1}$ were normalized by plaque forming units (PFU) $\mathrm{ml}^{-1}$ and represented as the TrU of an average phage titer (1E+9 PFU). $\operatorname{TrU~} \mathrm{ml}^{-1}$ for $\Delta$ terS were $<10$. Values are means \pm SD ( $n=3$ independent samples). 

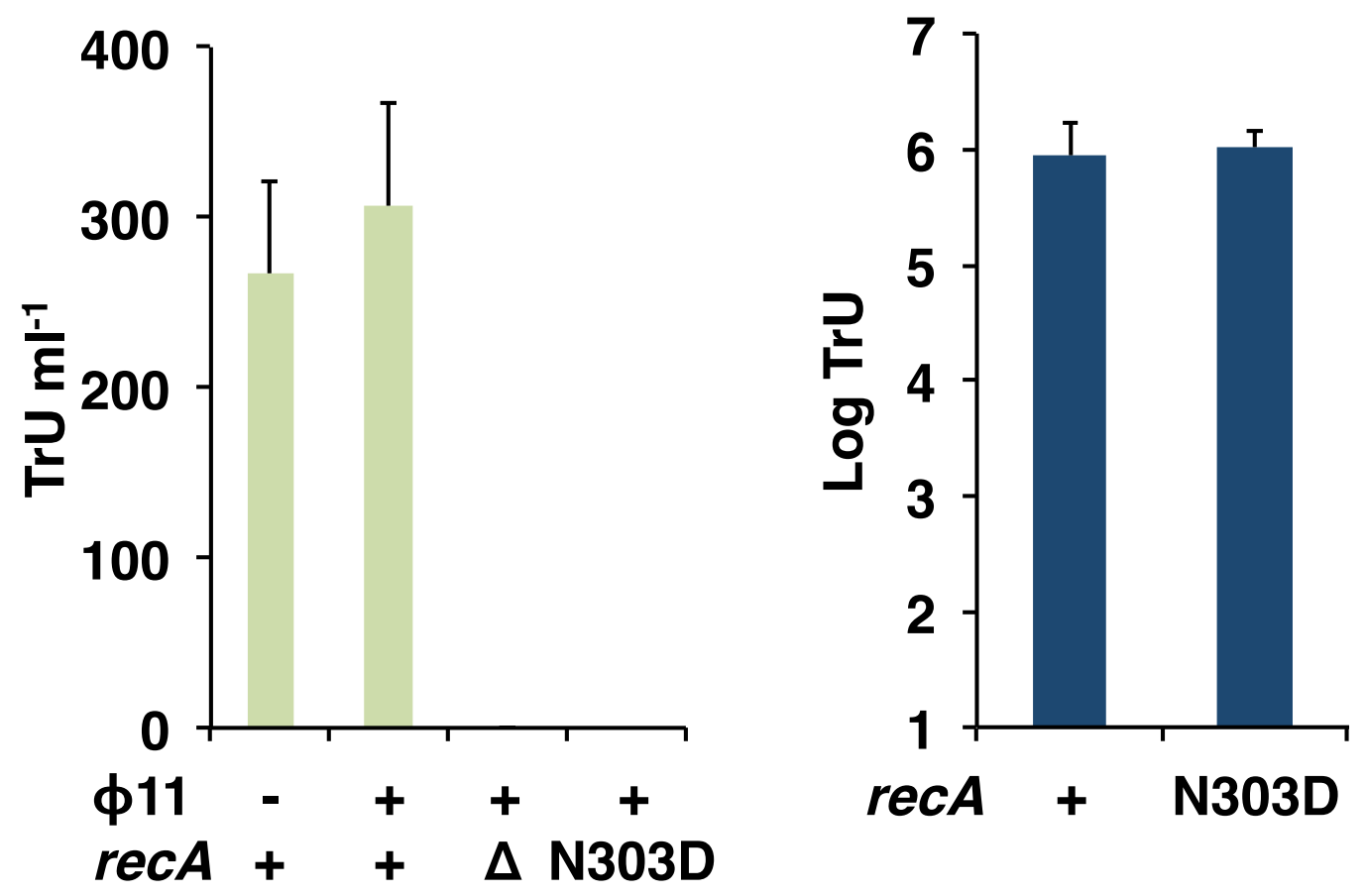

Figure S7. Recombination does not play a role in the generation of transducing particles by lateral transduction.

(A) Mutants of recA tested for recombination competence. Strains were used as recipients for an $80 \alpha$ lysate containing agr::ars $A B C$ for recombination. The results are represented as transduction units (TrU) $\mathrm{ml}^{-1}$. (B) $\phi 11$ lysogen induction tested for transfer of a $\mathrm{Cd}^{\mathrm{R}} 5 \mathrm{~kb}$ downstream of the $a t t B_{\phi 11}$ to $S$. aureus. Strains lysogenic (+) or non-lysogenic (-) for $\phi 11$ are indicated. The $\operatorname{rec} A$ genotypes are wild type $(+)$, deletion $(\Delta)$, and $\operatorname{rec} A(\mathrm{~N} 303 \mathrm{D})$. TrU $\mathrm{ml}^{-1}$ were normalized by plaque forming units (PFU) $\mathrm{ml}^{-1}$ and represented as the TrU of an average phage titer (1E+9 PFU). Values are means \pm SD ( $n=3$ independent samples).

A recA (N303D) mutant is defective for recombination but not for LexA cleavage and the SOS response. This mutant was necessary because a $\triangle r e c A$ null mutant is defective for the SOS response and unable to induce the prophage and produce a phage lysate. Shown in Fig. S7A, $\phi 11$ lysogens with $\triangle \operatorname{rec} A$ null or a $\operatorname{rec} A(\mathrm{~N} 303 \mathrm{D})$ allele were both completely defective for recombination when used as recipients for GT of an agr loci (accessory gene regulator) marked with an arsenite resistance cassette. However, lysates from $\phi 11$ induction in a wt or $\operatorname{rec} A(\mathrm{~N} 303 \mathrm{D})$ background transferred the $\mathrm{Cd}^{\mathrm{R}}{ }_{1}$ marker at comparable frequencies (Fig. S7B), showing that recombination is not involved in $\mathrm{Cd}^{\mathrm{R}}{ }_{1}$ packaging. 
A

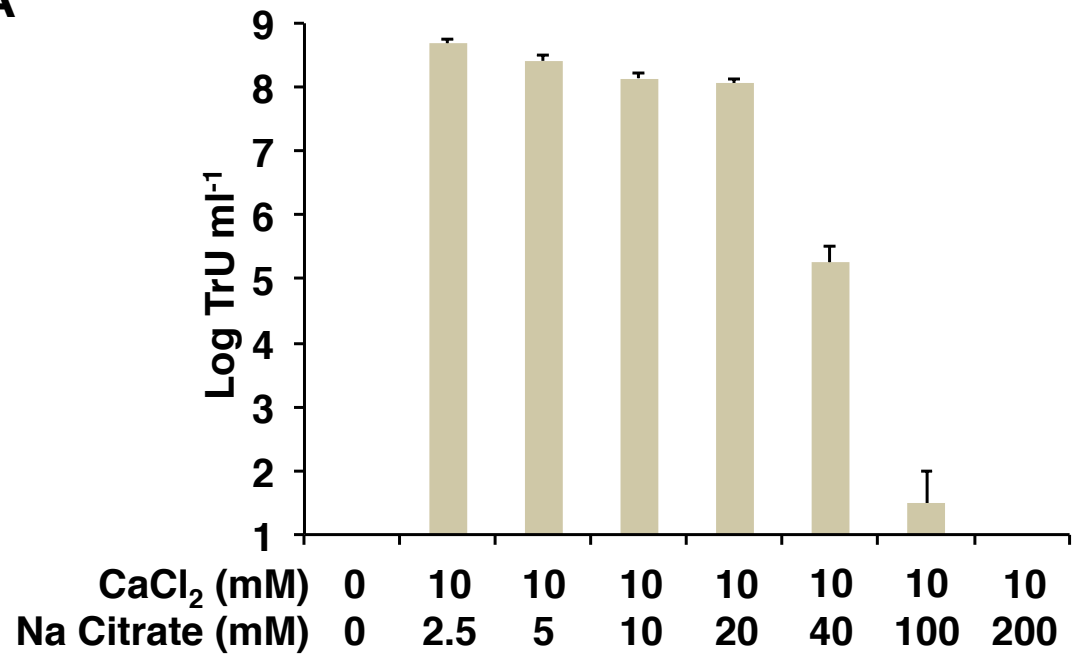

B

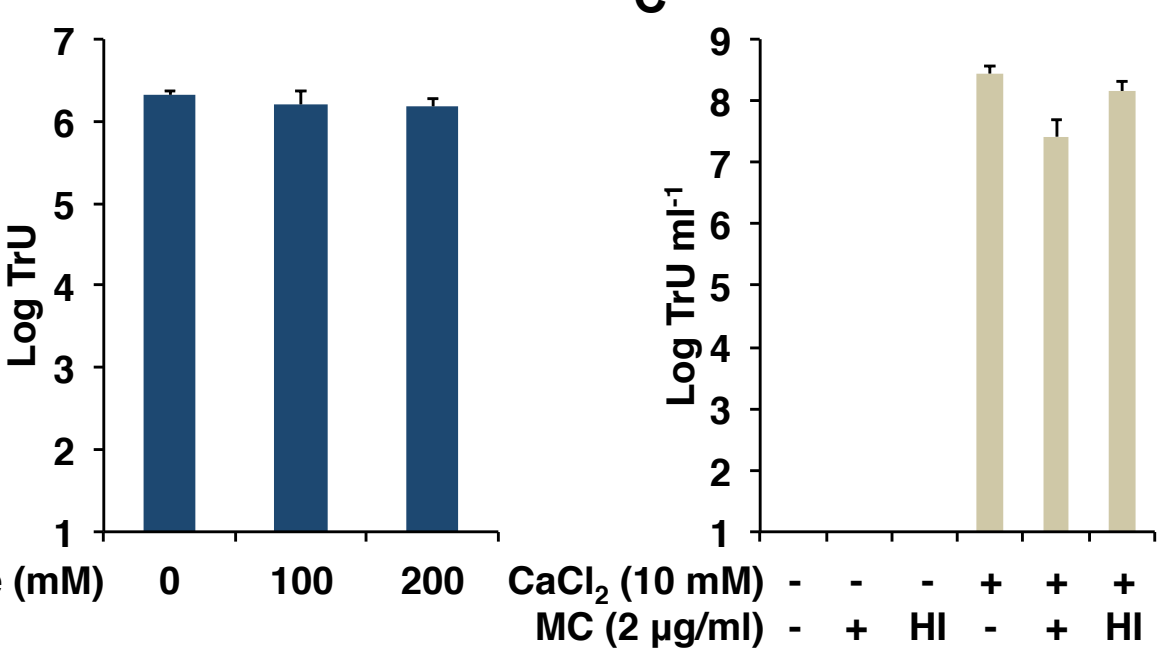

Figure S8. Super-infection does not play a role in lateral transduction.

(A) Determination of optimal concentration of chelating agent, $\mathrm{Na}$ citrate, for complete inhibition of phage adsorption. $\mathrm{CaCl}_{2}$ is required for staphylococcal phage adsorption and $10 \mathrm{mM} \mathrm{CaCl}$ is standardly used for transductions. Increasing concentrations of $\mathrm{Na}$ citrate were added to $\mathrm{S}$. aureus cells in $10 \mathrm{mM} \mathrm{CaCl}$ and infected with an $80 \alpha-S a P I 2$ (tsst1::tetM) lysate. The results are represented as transduction units (TrU) $\mathrm{ml}^{-1}$. (B) $\phi 11$ lysogens were induced with mitomycin C (MC) in the presence of $100 \mathrm{mM}$ or $200 \mathrm{mM}$ $\mathrm{Na}$ citrate and tested for transfer of a $\mathrm{Cd}^{\mathrm{R}} 5 \mathrm{~kb}$ downstream of the attB $B_{\phi 11}$ to $\mathrm{S}$. aureus. $\mathrm{TrU} \mathrm{ml}^{-1}$ were normalized by plaque forming units (PFU) $\mathrm{ml}^{-1}$ and represented as the TrU of an average phage titer (1E+9 PFU). (C) $S$. aureus cells with or without $10 \mathrm{mM} \mathrm{CaCl}_{2}$ and mitomycin $\mathrm{C}(+)$ or heat inactivated $\mathrm{MC}$ (HI) were infected with an 80a-SaPI2 (tsst1::tetM) lysate. The results are represented as $\mathrm{TrU} \mathrm{ml}^{-1}$. Values are means \pm SD ( $n=3$ independent samples).

Staphylococcal phages require divalent cations for adsorption to host cells, and the induction media contains only tryptic soy broth (TSB) and mitomycin C. To confirm that TSB is not able to support phage adsorption, we made use of a $S$. aureus pathogenicity island (SaPI) marked with a tetracycline resistance marker that is specially packaged by $80 \alpha$ and transferred at high frequencies. Fig. S8A shows that in the presence of $10 \mathrm{mM} \mathrm{CaCl}_{2}, \mathrm{SaPI} 2$ transferred at a frequency between $1 \mathrm{E}+8$ to $1 \mathrm{E}+9 \mathrm{TrU}$, but was not detectable without $\mathrm{CaCl}_{2}$, showing that phage adsorption is unlikely under the conditions of prophage induction. Furthermore, we induced $\phi 11$ in the presence of sodium citrate at concentrations (100 or $200 \mathrm{mM}$ ) sufficient to chelate $10 \mathrm{mM} \mathrm{CaCl}_{2}$ and block phage adsorption (Fig. S8B), and showed that the packaging and transfer frequencies of the $\mathrm{Cd}^{\mathrm{R}}{ }_{1}$ marker were unaffected. Lastly, we tested mitomycin $\mathrm{C}$ and heat-inactivated mitomycin $\mathrm{C}$ to determine if there was a contamination of divalent cation and found that SaPI2 transfer was not detectable with mitomycin $\mathrm{C}$ and heat-inactivated mitomycin $\mathrm{C}$ unless $\mathrm{CaCl}_{2}$ was added (Fig. S8C). 


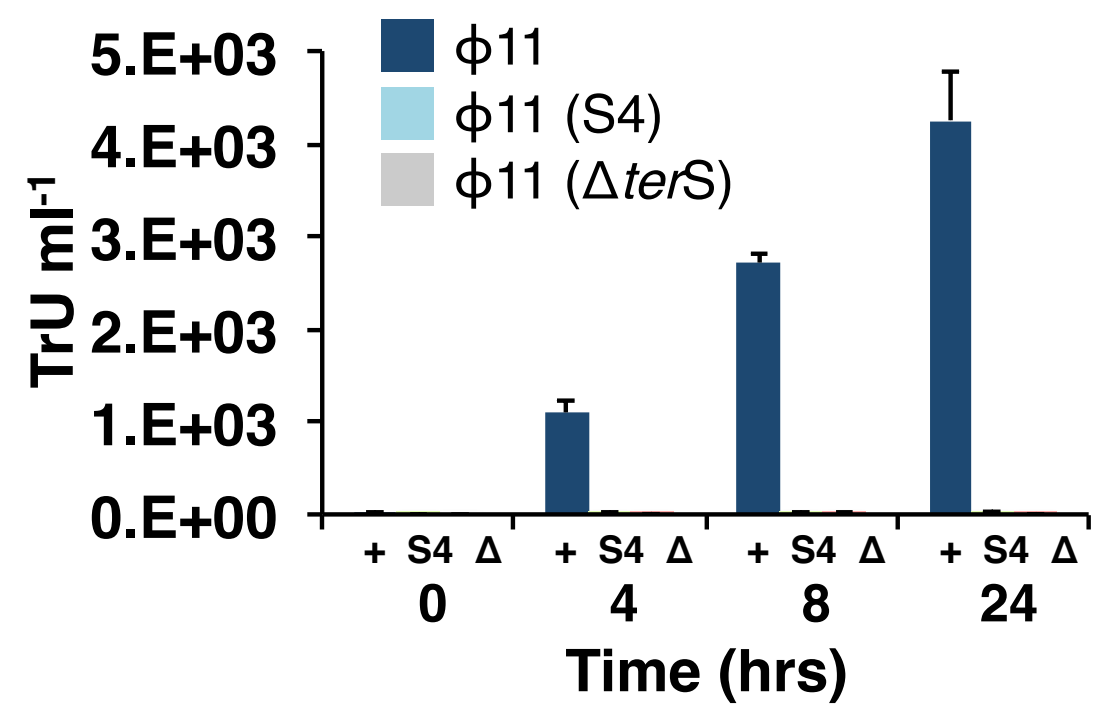

Figure S9. Spontaneous lateral transduction.

$\phi 11$ lysogenic strains were mixed with a streptomycin-resistant $S$. aureus recipient and tested for transfer of the $\mathrm{Cd}^{\mathrm{R}}{ }_{2}$ marker. Strains were left to induce spontaneously, without UV or chemical induction. Genotypes of lysogens are indicated as wild type $(+), \phi 11$ prophage integrated at the SaPI 4 attB (S4), and wild type $\$ 11$ prophage carrying a deletion in the terS gene $(\Delta)$. Transduction units $(\operatorname{TrU}) \mathrm{ml}^{-1}$ are represented as the equivalent of $1 \mathrm{ml}$ of $\mathrm{OD}_{600}=$ 1.0 to adjust for minor growth. Values are means $\pm \mathrm{SD}$ ( $n=3$ independent samples). 


\section{oriC}

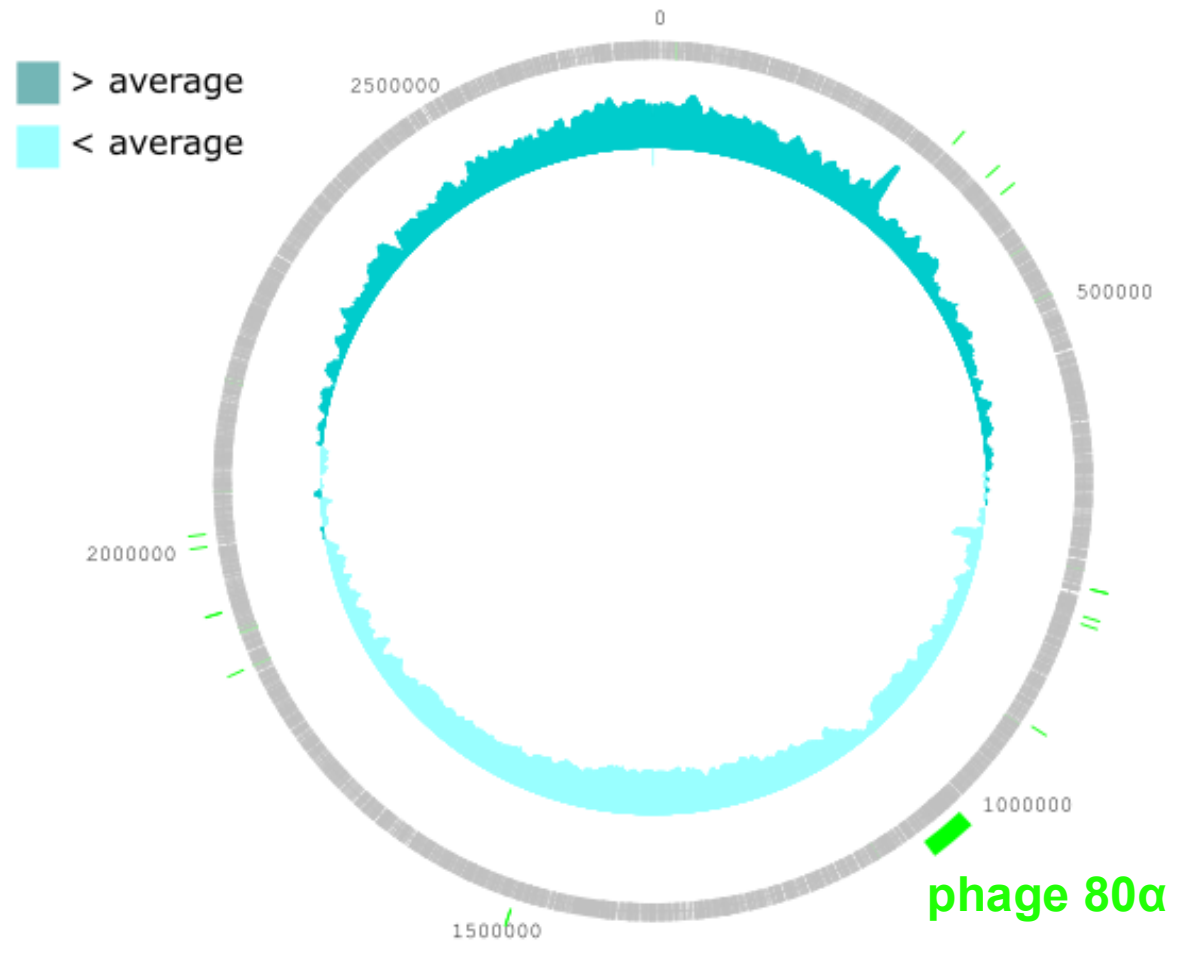

Figure S10. Whole genome sequencing and DNA quantification after induction of an $80 a$ lysogen.

DNA coverage decreases linearly from the oriC origin of replication towards the terminus. The degree coverage greater than the average of the entire chromosome is represented in teal. The degree coverage lesser than the average of the entire chromosome is represented in turquoise. Hence, the region furthest from the oriC displays the least DNA coverage and the greatest teal. Induction of the $80 \alpha$ prophage results in a large amplification of the viral genome, hence the reduction in lesser than the average turquoise at the $80 \alpha$ prophage site. 
A Phages $\phi N M 1$ \& $\phi N M 3$

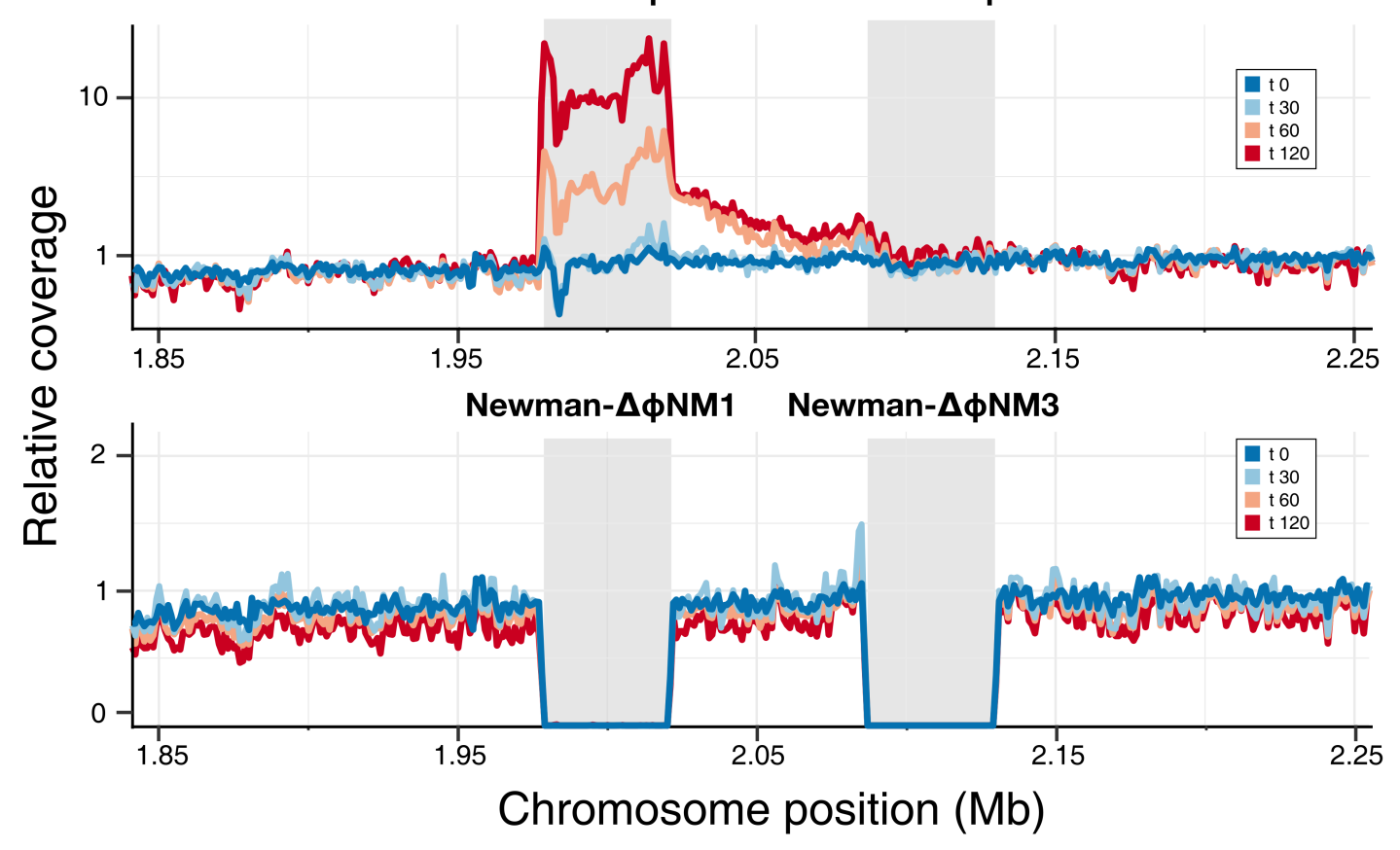

\section{B Phage $\phi N M 2$}

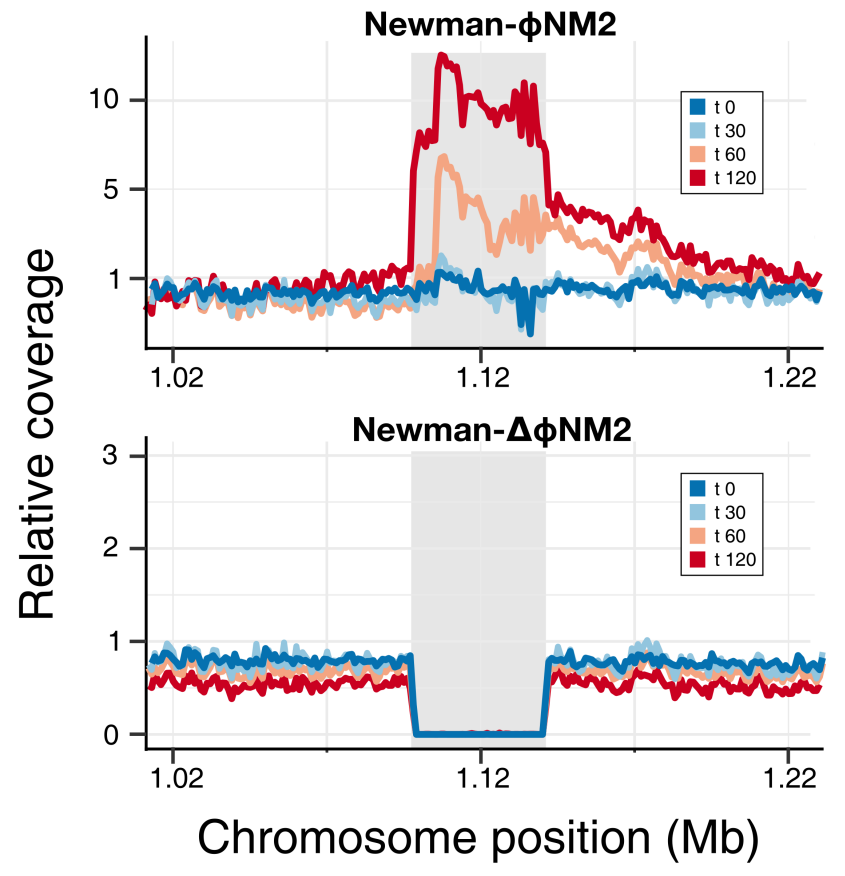

C Phage $\phi N M 4$

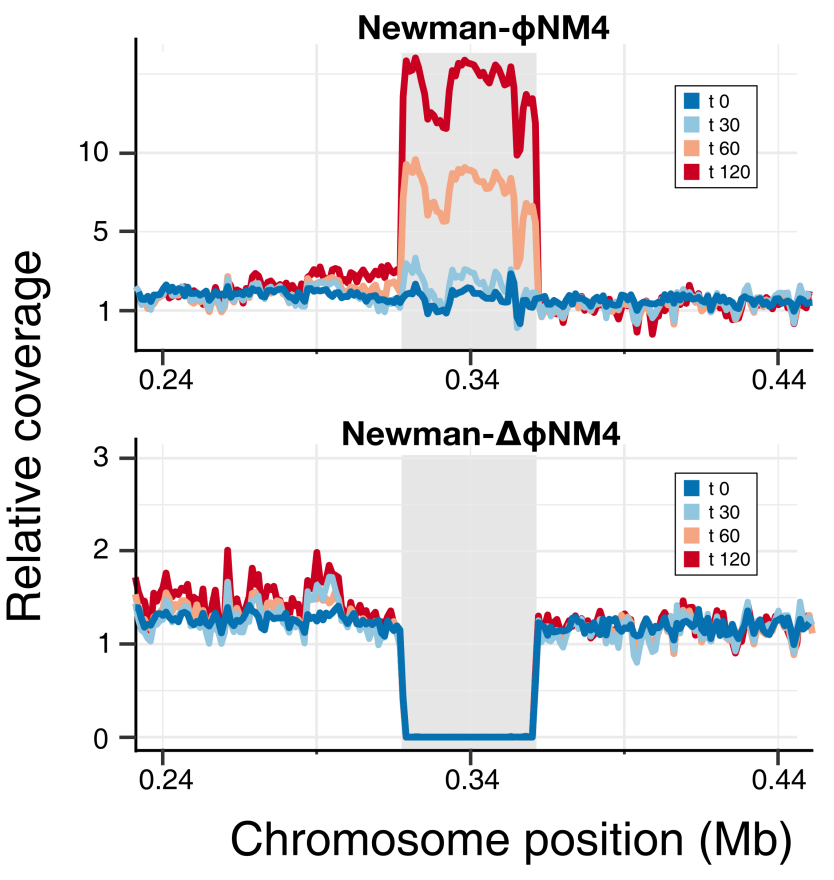

Figure S11. Staphylococcal phages replicate in situ before excision.

Relative abundance of phage genomic DNA and the chromosomal regions proximal to where they integrate for (A) $\phi N M 1$ and $\phi N M 3,(B) \phi N M 2$, and (C) $\phi N M 4$. Samples were analysed at 0 (blue), 30 (light blue), 60 (pink) and 120 minutes (red) post-induction with mitomycin C. Shaded grey rectangles represent the location of the prophages in the S. aureus chromosome. 


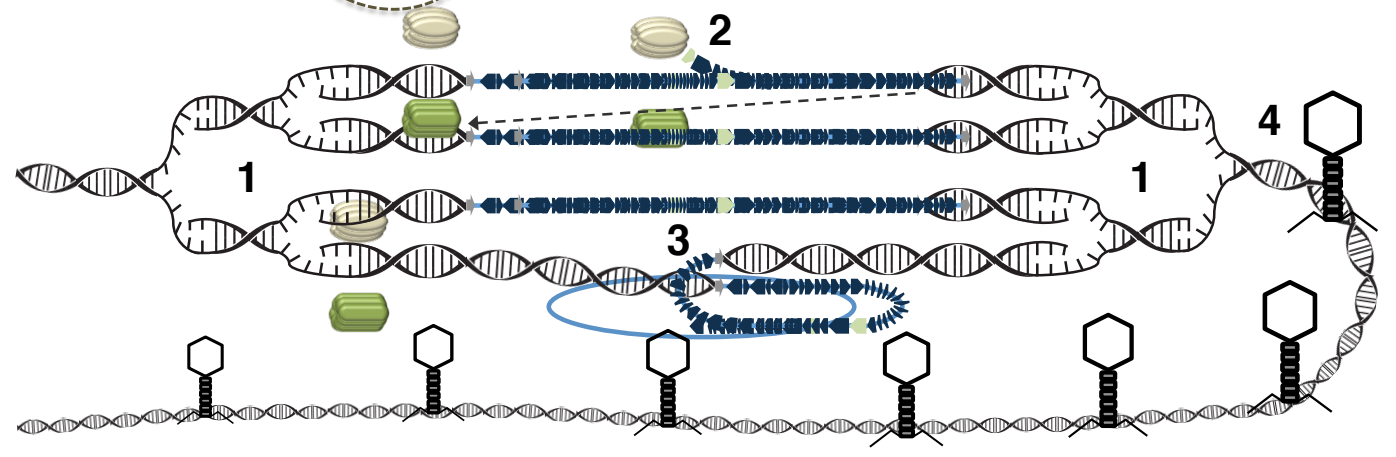

Figure S12. Model for lateral transduction.

The induced prophage genome (blue) replicates in situ bidirectionally (1) and amplifies the adjacent bacterial DNA (gray). Phage terminase initiates in situ DNA packaging from a pac site (2), while prophage excision occurs (3) in parallel. Prophage excision produces infectious phage particles. Lateral transducing particles are completed (4) when a headful capacity is reached. The DNA packaging machinery then proceeds to package additional headfuls of bacterial chromosome for several hundred kilobases. An arrow indicates a possible intramolecular integration joining two prophages that could result in an infectious particle by in situ packaging. 


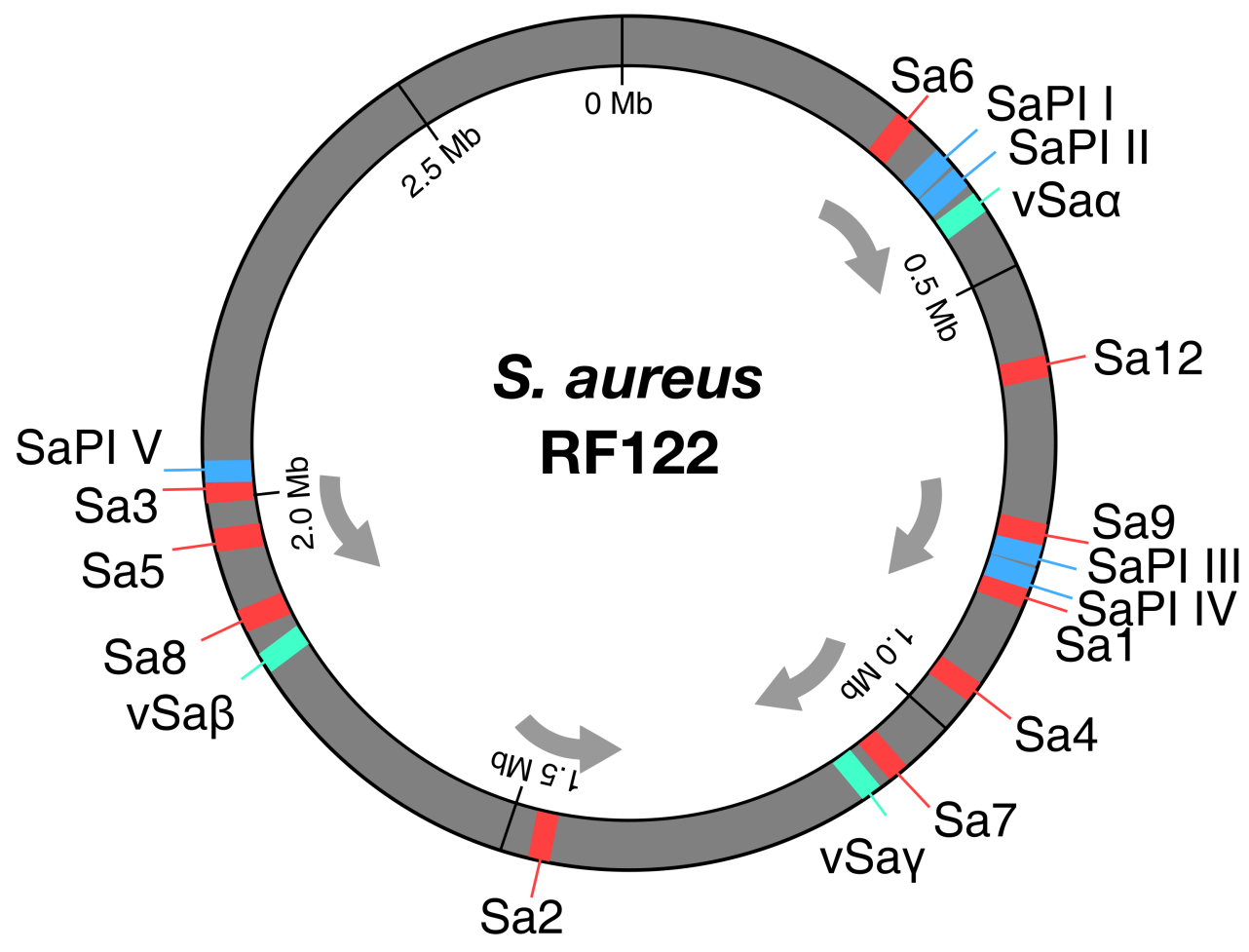

Phage $\square$ Pathogenicity
island

Figure S13. Lateral transduction influences genomic architecture.

The common attB sites of staphylococcal phages and pathogenicity islands mapped on to the genome of $S$. aureus strain RF122. Phage packaging directionality is determined by the orientation of the terS gene. 


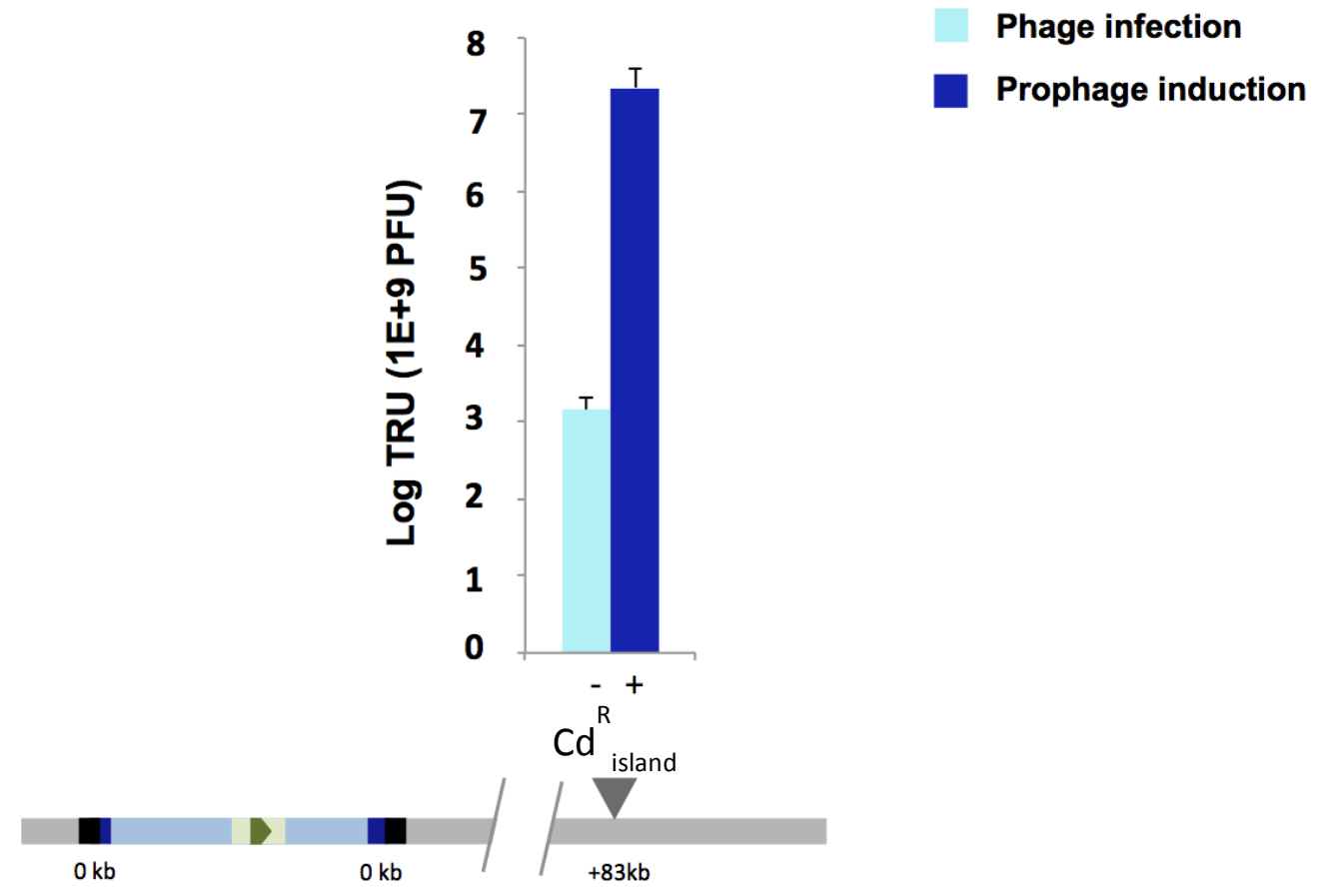

Figure S14. High frequency transfer of vSaa by lateral transduction.

Transfer of the chromosomal vSaa island after $\$ 52 \mathrm{~A}$ infection or lysogen induction. Nonlysogenic strains (light blue) were infected with $\phi 52 \mathrm{~A}$ or the lysogenic $\phi 52 \mathrm{~A}$ wt (dark blue) strains were induced with mitomycin $\mathrm{C}$ and the lysates tested for transduction into $\mathrm{S}$. aureus. The $\mathrm{Cd}^{\mathrm{R}}$ marker is shown inserted into the vSaa island. Transduction units (TrU) $\mathrm{ml}^{-1}$ were normalized by plaque forming (PFU) units $\mathrm{ml}^{-1}$ and represented as the TrU of an average phage titer (1E+9 PFU). Values are means $\pm \mathrm{SD}(n=3$ independent samples). 




Figure S15. Genomic comparisons of the Sa6 phage insertion site flanking region.

Analysis of gene conservation by classifying clusters of homologous gene families using the algorithm OrthoMCL, marking the genes based on their degree of conservation. Genes located on the left side of the attB site are more conserved than those at the right. The dark blue genes found downstream the attB site correspond with the SaPI attB site type I, SaPI site type II and vSaa islands. 


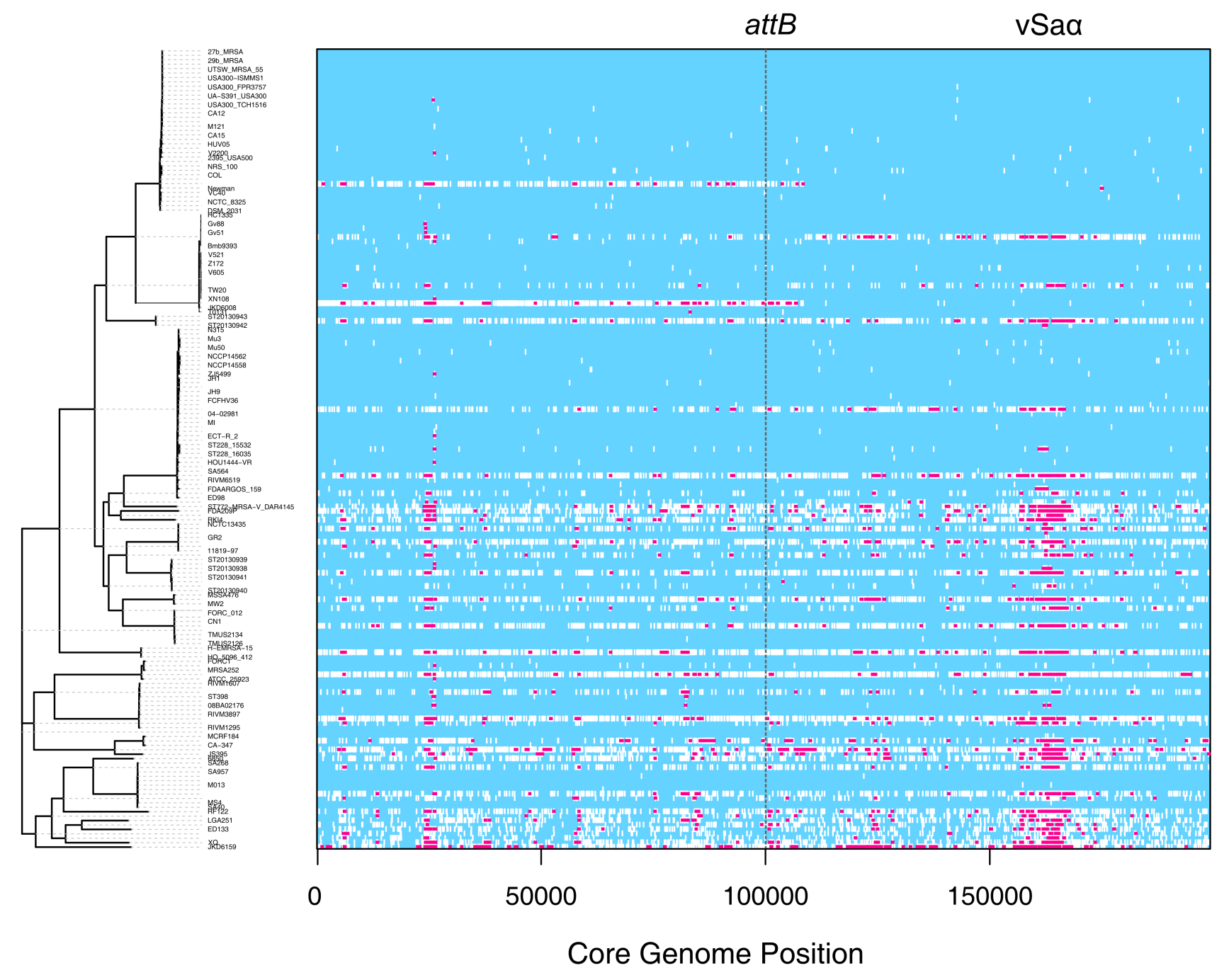

Figure S16. ClonalFrameML analysis of recombination of the Sa6 attB flanking region on the $200 \mathrm{~Kb}$ conserved across $101 \mathrm{~S}$. aureus genomes.

The central line represents the attB related gene, the recombination events (red) were more frequent and much longer downstream the attB site (right side). 1169 recombination events were detected on 70 branches of the $S$. aureus phylogeny, with multiple importations of up to $3.7 \mathrm{~Kb}$ identified $60 \mathrm{~Kb}$ downstream the attB site (vSaa), suggesting this region is a recombination hotspot. Although some recombination was also identified upstream the attB site, we found that the number of recombination events and the number of recombinant bases downstream the attB site were significantly higher compared to the recombination identified upstream this site, suggesting it is due to the mobile genetic elements present in that area. 


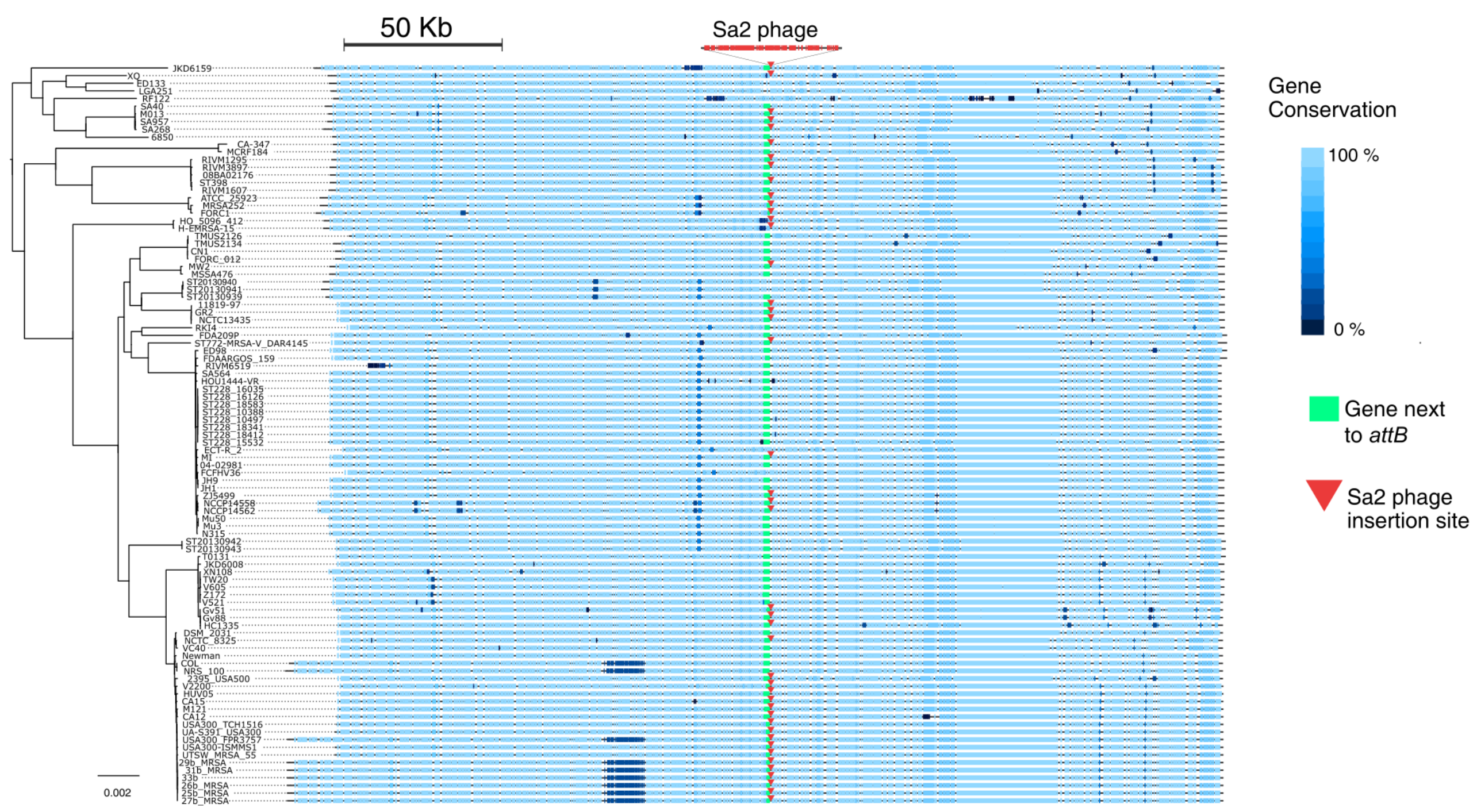

\section{Figure S17. Genomic comparisons of the Sa2 phage insertion site flanking regions.}

Analysis of gene conservation by classifying clusters of homologous gene families using the algorithm OrthoMCL, marking the genes based on their degree of conservation. Genes located on both the left and right side of the attB site are very conserved, but on the right side the highly recombinant ebh gene is present. 


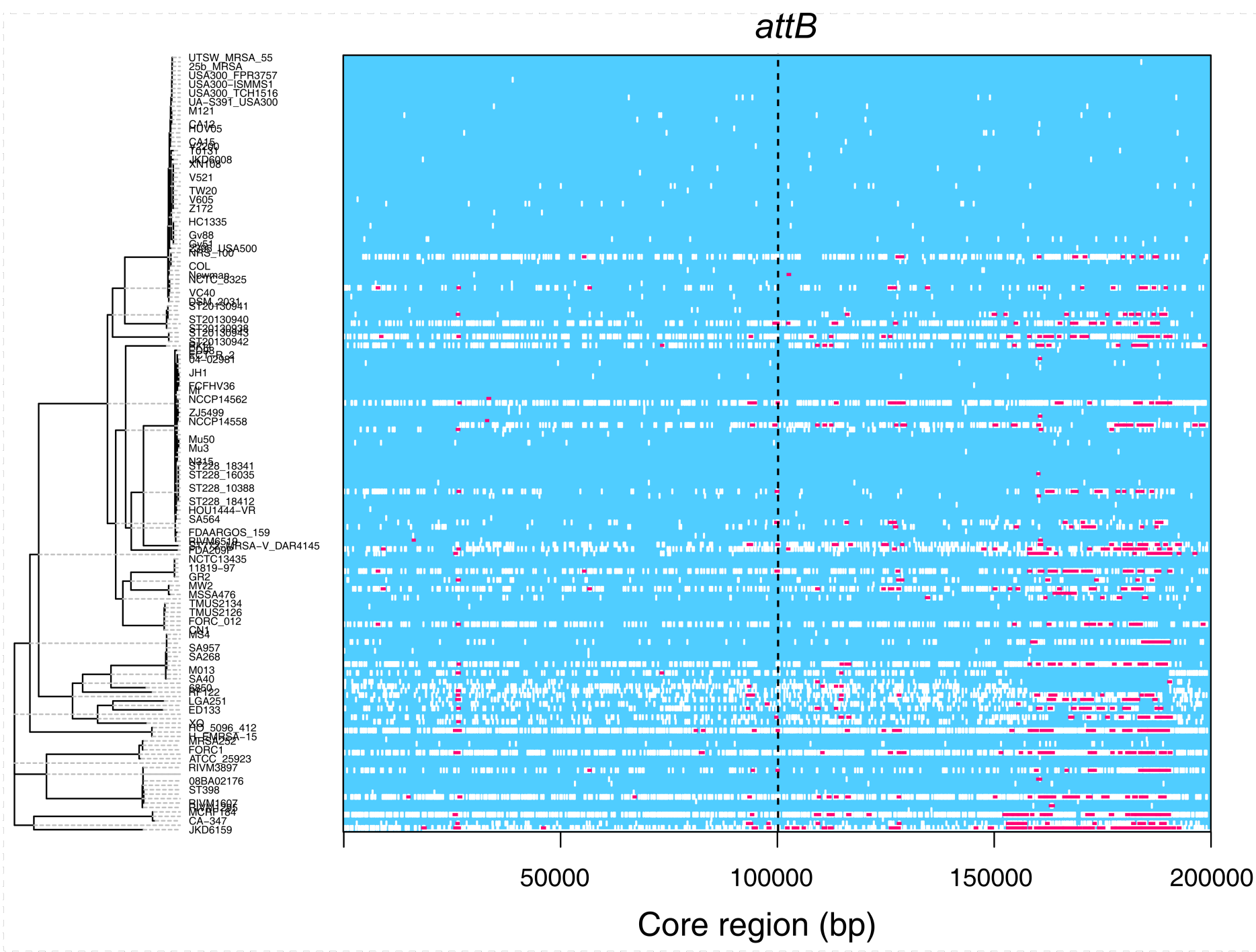

Figure S18. ClonalFrameML analysis of recombination of the Sa2 attB flanking region on the $200 \mathrm{~Kb}$ conserved across $101 \mathrm{~S}$. aureus genomes.

With the central line representing the attB site, recombination events (red) were more frequent and much longer downstream the attB site (right side), suggesting that this region is a recombination hotspot (paired Wilcoxon test for recombinant events $p$-value $=1.308 \mathrm{e}-08$, unpaired Wilcoxon test for recombinant nucleotides $p$-value $=5.514 \mathrm{e}-07$ ). This core genome hotspot corresponds with the ebh gene, encoding the surface giant protein Ebh. 
Table S1. Strains used in this study.

Table S2. Oligonucleotides used in this study.

Table S3. Plasmids used in this study.

Table S4. S. aureus strains used for the genomic analyses. 